\title{
Etiología de las Pérdidas Dentales en Pacientes Rehabilitados con Prótesis sobre Implantes
}

\author{
Etiology of Tooth Loss in Patients Rehabilitated with Dental Implants
}

\begin{abstract}
Guilherme da Rocha Scalzer Lopes; Jefferson David Melo de Matos²; Gabriel Pfister Sarcinelli Barbosa ${ }^{3}$; André Guimarães Rodrigues ${ }^{4}$; Renato Sussumu Nishioka5; Valdir Cabral Andrade ${ }^{6}$ \& Selva Maria Gonçalves Guerra ${ }^{7}$
\end{abstract}

LOPES, G. R. S.; MATOS, J. D. M.; BARBOSA, G. P. S.; RODRIGUES, A. G.; NISHIOKA, R. S.; ANDRADE, V. C. \& GUERRA, S. M. G. Etiología de las pérdidas dentales en pacientes rehabilitados con prótesis sobre implantes. Int. J. Odontostomat., 12(3):280-286, 2018.

RESUMEN: El objetivo de esta investigación fue analizar la etiología de las pérdidas dentales en pacientes rehabilitados con prótesis sobre implantes. Se realizó un estudio transversal con informaciones de 93 pacientes de una clínica privada que recibieron tratamiento con implantes en el período de 2000 a 2009. Los datos fueron recolectados a través de las fichas clínicas, documentaciones radiográficas y fotografías de los casos clínicos. Se levantaron datos sobre género, edad, nivel de escolaridad, hábito de fumar, tipo de diente extraído y razón de la extracción. Los resultados mostraron que la razón periodontal fue el principal motivo de las pérdidas dentales, representando el $24,7 \%$, seguida por las fracturas radiculares con pines metálicos $(23,7 \%$ ) y caries secundarias $(15,1 \%)$. En los pacientes menores de 40 años, los traumatismos dentales y las fracturas radiculares con pino fueron responsables de la mayoría de las pérdidas. Las razones de las pérdidas dentales mostraron significancia estadística con la variable edad $(p<0,05)$. El género, el hábito de fumar y el grado de escolaridad no mostraron asociación significativa $(p>0,05)$ con las razones de las pérdidas dentales.

PALABRAS CLAVE: etiologia, implantes dentales, pérdida de diente.

\section{INTRODUCCIÓN}

Diversos recursos odontológicos posibilitan la manutención del elemento dental en función (Sardinha et al., 2006), aun así, la perdida de los dientes es un problema de salud pública en Brasil y en el mundo (Caldas Junior et al., 2003). Muchos dientes continúan siendo extraídos y la toma de decisión en cuanto al mantenimiento o no del paciente dental es influenciada por varios factores (Bahrami et al., 2008). Los procedimientos restauradores pueden posponer la pérdida del elemento dental, pero en esas pérdidas pueden ocurrir a mediano y largo plazo, principalmente cuando estos pacientes necesitan un tratamiento multidisciplinario y un acompañamiento profesional (Ricucci \& Grosso, 2006).

Hace algunas décadas, los implantes ya se consideraron una gran opción de tratamiento en la sustitución de dientes perdidos por diferentes factores etiológicos (Bailit, 1987; Braun, 1987). En la actualidad, la utilización de implantes presenta gran previsibilidad, además de buenos resultados en el restablecimiento estético y funcional (Roccuzzo et al., 2014; Rokn et al., 2017); con ello se cuestiona la decisión de mantener los elementos dentales que

\footnotetext{
${ }^{1}$ Programa de Doctorado, Departamento de Prostodoncia, Universidade Estadual Paulista Júlio de Mesquita Filho UNESP, São José dos Campos - SP, Brasil.

${ }^{2}$ Programa de Maestría, Departamento de Prostodoncia, Universidade Estadual Paulista Júlio de Mesquita Filho UNESP, São José dos Campos - SP, Brasil.

${ }^{3}$ Maestría en Clínica Odontológica, Departamento de Odontología, Universidade Federal de Espírito Santo UFES, Vitória - ES, Brasil.

${ }^{4}$ Medicina por la Universidad Maria Serrana, Asunción - Paraguay.

${ }^{5}$ Departamento de Odontología, Universidade Estadual Paulista Júlio de Mesquita Filho UNESP, São José dos Campos - SP, Brasil.

${ }^{6}$ Departamento de Odontología, Universidade Federal de Juiz de Fora UFJF, Governador Valadares - MG, Brasil.

${ }^{7}$ Departamento de Odontología, Universidade Federal de Espírito Santo UFES, Vitória - ES, Brasil.
} 
LOPES, G. R. S.; MATOS, J. D. M.; BARBOSA, G. P. S.; RODRIGUES, A. G.; NISHIOKA, R. S.; ANDRADE, V. C. \& GUERRA, S. M. G. Etiología de las pérdidas dentales en pacientes rehabilitados con prótesis sobre implantes. Int. J. Odontostomat., 12(3):280-286, 2018.

presenten un alto grado de compromiso o aquellos que necesiten procedimientos restauradores largos y complejos (Ricucci \& Grosso).

Sin embargo, para lograr éxito en el tratamiento con prótesis sobre implante es fundamental una planificación adecuada, incluyendo la investigación del historial del paciente, ya que estos diferentes factores etiológicos pueden influir en el pronóstico del tratamiento con implantes, por ejemplo, los pacientes que hayan perdido dientes por enfermedad periodontal pueden presentar un mayor riesgo de desarrollar enfermedades peri-implantarias (Klinge et al., 2005; Lindhe et al., 2008; Meyle, 2008; HeitzMayfield, 2008; Cho-Yan Lee et al., 2011) (en el caso de las mujeres) (Sgolastra et al., 2015).

Los estudios que involucran la etiología de la pérdida dental en pacientes que recibieron prótesis sobre implantes son escasos, lo que indica la necesidad de un mejor entendimiento, tanto para la prevención de nuevas pérdidas dentales, como para el éxito del tratamiento rehabilitador con implantes. Aunque el historial de la pérdida dental puede presentarse como factor de riesgo, aún no hay consenso en cuanto a su influencia en el pronóstico de la terapia con implantes a largo plazo (Sgolastra et al.). Además, la prevención de nuevas pérdidas dentales es fundamental también en la implantologia, pues cuanto mayor es el número de dientes perdidos más desafiante y compleja es la rehabilitación del paciente (Bahrami et al.).

Siendo así, el conocimiento de las razones por las que se pierden los dientes permanentes, en este caso en pacientes con acceso a servicios odontológicos particulares, se hace necesario a medida que el acompañamiento profesional y los tratamientos odontológicos previos no fueron capaces de evitar las pérdidas dentales. En función de la necesidad de información sobre la etiología de las pérdidas dentales en pacientes rehabilitados con implantes, este estudio se propone aportar datos sobre estas pérdidas.

\section{MATERIAL Y MÉTODO}

Para la realización de este estúdio, el proyecto de investigación fue sometido al Comité de Ética en Investigación del Centro de Ciencias de la Salud de la Universidad Federal de Espírito Santo, siendo aprobado el 18/12/2008 bajo el $N^{\circ} 073 / 08$. Este trabajo se trata de un estudio analítico, observacional con un delineamiento transversal.

Para la recolección de datos se empleó un instrumento denominado "ficha de recolección de datos", con el objetivo de catalogar datos de prontuarios de pacientes que se sometieron a la extracción dental para instalación de implantes, entre los años 2000 y 2009, en una clínica privada en el municipio de Vitória-ES, siendo atendidos por una misma equipo de profesionales.

La recolección de las informaciones fue realizada a ciegas por un investigador no involucrado en las etapas quirúrgicas y/o protéticas del tratamiento, a través del acceso a los prontuarios, documentación radiográfica y fotografías de los casos clínicos. Después de la recogida de datos de los prontuarios, a partir de una muestra de conveniencia, un total de 93 individuos antedió los criterios de inclusión, presentando un total de 209 dientes extraídos para la instalación de implantes.

En esta etapa, se realizó un análisis descriptivo, clasificando los dientes siguiendo la notación dental de la Federación Dentária Internacional (FDI) en 28 códigos (excluidos los terceros molares para la investigación) de acuerdo con la razón de la pérdida presentada (Tabla I).

Con el fin de utilizar el individuo como unidad de muestreo y mejorar la investigación clínica del presente trabajo se realizaron elecciones aleatorias de un elemento dental perdido por paciente, totalizando 93 elementos dentales, buscando resaltar la importancia de la variación individual en la unidad estadísti-

Tabla I. Notación dental de la Federación Dental Internacional.

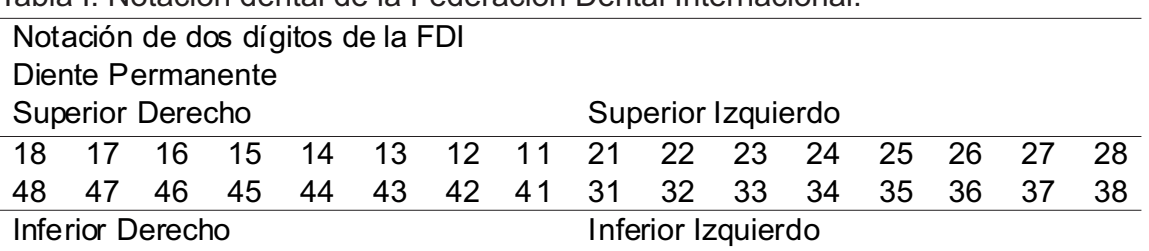


ca. Se incluyeron en este estudio pacientes que aceptaron participar en la investigación firmando el Término de Consentimiento Libre y Esclarecido, individuos de ambos sexos, mayores de 18 años y que se sometieron a la extracción dental para la instalación de implantes entre los años 2000 y 2009. fueron excluidos pacientes que no se ajustaban a los criterios de inclusión y pacientes cuya razón de la pérdida dental no fue identificada y/o con fichas clínicas con datos ausentes.

Las siguientes variables consideradas en esta investigación fueron: a) características sociodemográficas: Datos relativos al género, edad, nivel de escolaridad, tipo de diente extraído, y uso del tabaco se correlacionaron. Estas variables se clasificaron según los datos contenidos en las fichas clínicas utilizadas por el consultorio para la evaluación de sus pacientes. Los individuos fueron divididos en dos grupos según la edad, un grupo formado por pacientes con edades entre 18 y 39 años y el otro 40 año o más, esta división fue utilizada para facilitar el análisis estadístico y la comparación con otros estudios. El nivel de escolaridad fue clasificado en: enseñanza primaria, secundaria incompleta, enseñanza media completa, enseñanza superior incompleta y enseñanza superior completa. b) clasificación dental: Los dientes se han agrupado de acuerdo con el tipo de diente y su ubicación en la arcada dental.

La clasificación de las pérdidas dentales fue realizada de acuerdo con el trabajo de Sulzer et al. (2004), siendo ellas: a) fractura vertical radicular - dientes extraídos por motivo de fracturas verticales - dientes fracturados, tratados endodónticamente y restaurados con pines y núcleos intra-radiculares metálicos fundidos o prefabricados; y dientes fracturados, tratados o no endodónticamente, restaurados o no sin la presencia de núcleos o pines metálicos. b) fracaso endodóntico - dientes extraídos por fracasos endodónticos y / o lesiones periapicales; c) reabsorción radicular - dientes extraídos debido a reabsorciones radiculares externas o internas debido a traumatismos dentales anteriores, tratamientos ortodónticos mal conducidos u otros motivos; d) traumatismo dental - dientes perdidos por traumatismos dentales directos (avulsiones y fracturas dentales extensas), incluyendo dientes perdidos por fracturas de los maxilares; e) caries primarias - dientes perdidos por caries extensas sin tratamiento y que imposibilitaron restauraciones adecuadas del elemento dental; f) caries secundarias - dientes extraídos por caries recurrentes en dientes previamente restaurados, incluyendo todo tipo de restauraciones y materiales restauradores, caries recurrentes que imposibilitar el mantenimiento del elemento dental; g) enfermedad periodontal - dientes perdidos por problemas periodontales y h) otros - extracciones por razones no englobadas en otras categorías.

Todas las variables relativas al implante se analizaron estadísticamente y, según el tipo de la variable a ser probada (categórica o numérica), se aplicó la prueba estadística más indicada. Se realizó un análisis descriptivo de los datos por medio de tablas de frecuencia con número porcentual para cada uno de los ítems de la investigación. La prueba Chi-cuadrado se utilizó para verificar la independencia entre las variables. Las pruebas se realizaron en el paquete estadístico - Social Package Statistical Science 15 (SPSS). El nivel de significancia establecido para las pruebas fue igual al $5 \%$, lo que estableció un intervalo de confianza del $95 \%$ para los resultados presentados.

\section{RESULTADOS}

La muestra fue constituida por 93 pacientes, siendo 30 pacientes $(32,3 \%)$ del género masculino y 63 pacientes $(67,7 \%)$ del género femenino, de éstos, 73 pacientes $(78,5 \%)$ presentaban edad por encima de los 40 años $78,5 \%$ ) (Tabla II)

Tabla II. Distribución de pacientes con pérdidas dentales sometidos a tratamientos con implantes según género y edad, Vitória-ES, 2009.

\begin{tabular}{lll}
\hline & Número & $\%$ \\
\hline Sexo & & \\
Masculino & 30 & 32,3 \\
Femenino & 63 & 67,7 \\
Franja etaria & & \\
18 a 39 años & 20 & 21,5 \\
40 años o mas & 73 & 78,5 \\
\hline Total & 93 & 100,0 \\
\hline
\end{tabular}

El nivel de escolaridad presentado por los pacientes mostró que 66 pacientes $(71 \%)$, presentaron nivel superior $(71 \%)$, sólo 2 pacientes $(2,2 \%)$ no completaron la enseñanza media. El hábito de fumar fue presentado por 11 pacientes (11,8\%) (Tabla III).

Los resultados demostraron que el principal motivo de las pérdidas dentales fue la enfermedad periodontal $(24,7 \%)$, seguida por la fractura vertical radicular con pino $(23,7 \%)$, caries secundaria $(15,1$ 
LOPES, G. R. S.; MATOS, J. D. M.; BARBOSA, G. P. S.; RODRIGUES, A. G.; NISHIOKA, R. S.; ANDRADE, V. C. \& GUERRA, S. M. G. Etiología de las pérdidas dentales en pacientes rehabilitados con prótesis sobre implantes. Int. J. Odontostomat., 12(3):280-286, 2018.

Tabla III. Caracterización de la muestra relacionada al nivel de escolaridad y hábito de fumar, Vitória-ES, 2009.

\begin{tabular}{lll}
\hline & Número & $\%$ \\
\hline Escolaridad & & \\
Enseñanza fundamental & 1 & 1,1 \\
Enseñanza media incompleto & 1 & 1,1 \\
Enseñanza media completo & 19 & 20,4 \\
Enseñanza superior incompleto & 6 & 6,5 \\
$\quad$ Enseñanza superior completo & 66 & 71,0 \\
Fumador & & \\
Si & 11 & 11,8 \\
No & 82 & 88,2 \\
\hline
\end{tabular}

$\%)$, fracaso endodóntico $(11,8 \%)$, reabsorción radicular $(9,7 \%)$, traumatismo dental $(7,5 \%)$, otros motivos $(4,3$ $\%)$ y fractura vertical radicular sin pino $(3,2 \%)$. Ningún paciente presentó pérdida dental por caries primaria (Tabla IV).
La distribución de las pérdidas dentales por grupo de dientes puede observarse en la (Tabla V). Se observa que el grupo de dientes más afectado por la pérdida fue el de los incisivos superiores $(35,5 \%)$, seguido por los molares inferiores $(19,4 \%)$ y premolares superiores $(17,2 \%)$. Estos tres grupos representaron el $72,1 \%$ de la muestra.

Las tablas cruzadas con el teste qui-cuadrado se utilizaron en el análisis representado en (Tabla VI). Una tabla cruzada muestra el número de casos en cada categoría, definida por dos o más grupos de variables categóricas. El test qui-cuadrado prueba la hipótesis de que las variables en una tabla cruzada son independientes. Un p-valor significativo $(<0,05)$ indica que hay alguna relación entre estas variables.

La intersección que mostró significancia estadística con los motivos de las pérdidas dentales fue el grupo de edad $(p=0,001)$.
Tabla IV. Principales razones de pérdidas dentales en pacientes rehabilitados con implantes, Vitória-ES, 2009.

\begin{tabular}{lcr}
\hline Razón de la pérdida & $\begin{array}{c}\text { Número de } \\
\text { dientes }\end{array}$ & $\%$ \\
\hline Fractura vertical radicular con pino & 22 & 23,7 \\
Fractura vertical radicular sin pino & 3 & 3,2 \\
Fracaso endodóntico & 11 & 11,8 \\
Reabsorción radicular & 9 & 9,7 \\
Traumatismo dental & 7 & 7,5 \\
Carie secundaria & 14 & 15,1 \\
Razón Periodontal & 23 & 24,7 \\
Otros & 4 & 4,3 \\
\hline TOTAL & 93 & 100,0
\end{tabular}

Tabla V. Distribución de los dientes perdidos por grupo de dientes en pacientes de clínica privada, Vitória-ES, 2009.

\begin{tabular}{lrr}
\hline Grupo de dientes & $\mathrm{N}$ & $\%$ \\
\hline Incisivos superiores & 33 & 35,5 \\
Caninos superiores & 4 & 4,3 \\
Pre-molares superiores & 16 & 17,2 \\
Molares superiores & 7 & 7,5 \\
Incisivos inferiores & 6 & 6,5 \\
Caninos inferiores & 1 & 1,1 \\
Pre-molares inferiores & 8 & 8,6 \\
Molares inferiores & 18 & 19,4 \\
Anteriores superiores & 37 & 39,8 \\
Posteriores superiores & 23 & 24,7 \\
Anteriores inferiores & 7 & 7,5 \\
Posteriores inferiores & 26 & 28,0 \\
\hline
\end{tabular}

\section{DISCUSIÓN}

La comparación entre investigaciones de razones de pérdidas dentales se ve dificultada por las diferentes metodologías utilizadas. En la presente investigación, el público objetivo se diferenció en el nivel de escolaridad y acceso a servicios odontológicos de la mayoría de las muestras poblacionales de los estudios epidemiológicos realizados. Por lo que se refiere a una investigación que involucra a pacientes con acceso a servicios odontológicos en una clínica particular, la clasificación utilizada no se basó en la propuesta por Kay \& Blinkhorn (1986), que se utiliza en muchos estudios epidemiológicos, principalmente por el hecho de las caries primarias y secundarias se incluyen en un mismo ítem y las secuelas de la caries, como la fractura radicular y el fracaso endodóntico no se clasifican por separado, siendo incluidas en las pérdidas por caries. Esta clasificación no demuestra con claridad los reales motivos de las pérdidas dentales, no permitiendo un análisis más detallado de los problemas que realmente llevan las pérdidas dentales en este grupo de pacientes.

Si la clasificación utilizada fue igualada, los resultados demostrarían la caries y sus secuelas como principal razón de las pérdidas den- 
LOPES, G. R. S.; MATOS, J. D. M.; BARBOSA, G. P. S.; RODRIGUES, A. G.; NISHIOKA, R. S.; ANDRADE, V. C. \& GUERRA, S. M. G. Etiología de las pérdidas dentales en pacientes rehabilitados con prótesis sobre implantes. Int. J. Odontostomat., 12(3):280-286, 2018.

Tabla VI. Cruzamiento entre las principales razones de las pérdidas dentales y otras variables en pacientes de clínica privada, Vitória-ES, 2009.

\begin{tabular}{|c|c|c|c|c|c|c|c|}
\hline \multirow[b]{2}{*}{ Variables } & \multirow[b]{2}{*}{$\begin{array}{l}\text { Fractura } \\
\text { radicular } \\
\text { con pino }\end{array}$} & \multicolumn{5}{|c|}{ Razón } & \multirow[b]{2}{*}{$p$-valor } \\
\hline & & $\begin{array}{c}\text { Fracaso } \\
\text { endodóntico }\end{array}$ & $\begin{array}{l}\text { Reabs. } \\
\text { Radicular }\end{array}$ & $\begin{array}{l}\text { Traumatismo } \\
\text { Dentario }\end{array}$ & $\begin{array}{c}\text { Carie } \\
\text { secundaria }\end{array}$ & $\begin{array}{l}\text { Razón } \\
\text { periodontal }\end{array}$ & \\
\hline \multicolumn{8}{|l|}{ Franja etaria } \\
\hline 18 a 39 años & $6(27,3)$ & $2(18,2)$ & $2(22,2)$ & $6(85,7)$ & $2(14,3)$ & $1(4,3)$ & 0,001 \\
\hline 40 años o más & $16(72,7)$ & $9(81,8)$ & $7(77,8)$ & $1(14,3)$ & $12(85,7)$ & $22(95,7)$ & \\
\hline \multicolumn{8}{|l|}{ Escolaridad } \\
\hline Fundamental & $0(0,0)$ & $0(0,0)$ & $0(0,0)$ & $0(0,0)$ & $1(7,1)$ & $0(0,0)$ & \\
\hline Medio incompleto & $0(0,0)$ & $0(0,0)$ & $0(0,0)$ & $0(0,0)$ & $1(7,1)$ & $0(0,0)$ & \\
\hline Medio completo & $5(22,7)$ & $1(9,1)$ & $1(11,1)$ & $3(42,9)$ & $2(14,3)$ & $6(26,1)$ & - \\
\hline Superior incompleto & $1(4,5)$ & $0(0,0)$ & $0(0,0)$ & $1(14,3)$ & $0(0,0)$ & $3(13,0)$ & \\
\hline Superior completo & $16(72,7)$ & $10(90,9)$ & $8(88,9)$ & $3(42,9)$ & $10(71,4)$ & $14(60,9)$ & \\
\hline \multicolumn{8}{|l|}{ Fumador } \\
\hline $\mathrm{Si}$ & $1(4,5)$ & $2(18,2)$ & $0(0,0)$ & $1(14,3)$ & $4(28,6)$ & $3(13,0)$ & 0,271 \\
\hline No & $21(95,5)$ & $9(81,8)$ & $9(100,0)$ & $6(85,7)$ & $10(71,4)$ & $20(87,0)$ & \\
\hline \multicolumn{8}{|l|}{ Sexo } \\
\hline Masculino & $6(27,3)$ & $3(27,3)$ & $4(44,4)$ & $4(57,1)$ & $2(14,3)$ & $10(43,5)$ & 0,292 \\
\hline Femenino & $16(72,7)$ & $8(72,7)$ & $5(55,6)$ & $3(42,9)$ & $12(85,7)$ & $13(56,5)$ & \\
\hline \multicolumn{8}{|l|}{ Ubicación } \\
\hline Anteriores superiores & $11(50,0)$ & $4(36,4)$ & $6(66,7)$ & $6(85,7)$ & $2(14,3)$ & $6(26,1)$ & \\
\hline Posteriores superiores & $8(36,4)$ & $1(9,1)$ & $1(11,1)$ & $1(14,3)$ & $6(42,9)$ & $4(17,4)$ & - \\
\hline Anteriores inferiores & $0(0,0)$ & $1(9,1)$ & $1(11,1)$ & $0(0,0)$ & $1(7,1)$ & $4(17,4)$ & \\
\hline Posteriores inferiores & $3(13,6)$ & $5(45,5)$ & $1(11,1)$ & $0(0,0)$ & $5(35,7)$ & $9(39,1)$ & \\
\hline
\end{tabular}

* p-valor $<0,050$.

tales, a continuación, aparecería la enfermedad periodontal. En el presente estudio se analizaron los resultados obtenidos en el estudio de las pérdidas dentales en diversos países (Cahen et al., 1985; Morita et al., 1994; Angelillo et al., 1996; Mccaul et al., 2001; JovinoSilveira et al., 2004, 2005). Sin embargo, esta clasificación ocultaría informaciones importantes de ser analizadas y discutidas, como fracturas radiculares de dientes con pines, caries secundarias e fracasos endodónticos. Información que puede hacernos reflexionar sobre tratamientos propuestos y realizados en el grupo de pacientes atendidos por servicios odontológicos privados. Además, un detalle de la clasificación nos permite un seguimiento futuro de los implantes colocados, evaluando la posible relación entre las causas de las pérdidas dentales y el éxito de los implantes.

La mayoría de los pacientes de la investigación (71 \%) presentó un nivel superior completo, lo que garantizó la homogeneidad en la muestra, una vez que el grado de escolaridad está relacionado al acceso a la información ya la atención médica, ya que estos factores influencian la salud del individuo como un todo. La muestra del estudio fue compuesta principalmente por pacientes del sexo femenino $(67,7 \%)$, con edad por encima de los 40 años (78,5\%). Esto no sólo retrata el aumento de la expectativa de vida de la población y de los cuidados con la salud, sino también la mayor búsqueda por tratamiento odontológico por el sexo femenino, según lo observado por (Lekholm et al., 1994; Karoussis et al., 2003; Swierkot et al., 2012).

La razón periodontal $(24,7 \%)$ fue el principal motivo de pérdidas dentales en la presente investigación. Esta constatación está de acuerdo con un estudio similar realizado por Sulzer et al. Este motivo de pérdida dental se observó principalmente en los pacientes mayores de 40 años. La pérdida por problemas periodontales en este grupo de edad se encuentra en otras investigaciones como la de Fardal et al. (2004) y puede ocurrir debido a la progresión de la pérdida de inserción con el avance de la edad (Tonetti et al., 2000; Fardal et al.). Y en el caso de las mujeres. En los pacientes con menos de 40 años los traumatismos dentales y las fracturas radiculares con pino fueron los principales responsables de las pérdidas. En este grupo de edad, hasta los 40 años, los resultados están de acuerdo con otros estudios que apuntan al traumatismo dental como principal causa de las pérdidas dentales (Andersson et al., 1995; Sulzer 
LOPES, G. R. S.; MATOS, J. D. M.; BARBOSA, G. P. S.; RODRIGUES, A. G.; NISHIOKA, R. S.; ANDRADE, V. C. \& GUERRA, S. M. G. Etiología de las pérdidas dentales en pacientes rehabilitados con prótesis sobre implantes. Int. J. Odontostomat., 12(3):280-286, 2018.

et al.), y corrobora con investigaciones sobre dientes que se ha convertido en una de las principales causas de accidentes automovilísticos (Melo et al., 2003), debido principalmente a las caídas y accidentes automovilísticos (Melo et al.).

Las fracturas radiculares de dientes con pines metálicos $(23,7 \%)$ fueron la segunda razón de extracciones más prevalente en la investigación. En la mayoría de los casos, la mayoría de las personas que sufren de depresión, que no se sienten atrapadas en el sistema nervioso. Pero los estudios previos demuestran que el uso de pines y núcleos metálicos puede aumentar el riesgo de pérdidas dentales (Willershausen et al., 2005; Wegner et al., 2006). Estos autores sugieren precaución cuando se indica el uso de los pines metálicos. La caries secundaria $(15,1$ \%) también apareció como una importante razón de exodontias. Por otro lado, ningún diente se ha perdido debido a la caries primaria. Estos hallazgos demuestran que los pacientes englobados en ese estudio presentaron a lo largo de la vida un acompañamiento odontológico y acceso a servicios odontológicos. Esta constatación de pérdidas por caries secundarias puede reflejar los efectos acumulativos de los defectos de las restauraciones a lo largo de la vida, llevando la pérdida dental en edades más avanzadas (JovinoSilveira et al., 2004).

En la mayoría de los casos, se observó un aumento de la incidencia de la enfermedad de Chagas, como las razones de las pérdidas dentales en la clínica privada. El grupo de dientes que presentaron mayor número de pérdidas fue el de los anteriores superiores $(39,8 \%)$. Este dato está de acuerdo con el estudio de Andersson et al. Esta constatación es justificada en la actual investigación por el alto porcentaje encontrado de dientes perdidos por fracturas radiculares y traumatismos dentales, que acometieron principalmente ese grupo de dientes, además, por tratarse de una zona estética, la rehabilitación con implantes es buscada más rápidamente por el paciente.

Las razones de las pérdidas dentales mostraron significancia estadística con la variable edad ( $p$ $<0,05)$. El género y el hábito de fumar no mostraron asociación significativa $(p>0,05)$ con las razones de las pérdidas en la clínica privada evaluada. Este resultado está en concordancia con el estudio de JovinoSilveira et al. (2004), donde la edad demostró significancia con las razones de las pérdidas. El hábito de fumar, en algunos estudios se muestra asociado a la enfermedad periodontal ya un aumento en el ries- go de pérdidas dentales (Axelsson et al., 1998), pero en el presente estudio la muestra de fumadores fue pequeña, por lo que las evaluaciones en ese ítem presentaron limitaciones.

\section{CONSIDERACIONES FINALES}

Puede concluirse a partir de este estudio que: La razón periodontal fue identificada como el principal motivo de pérdidas dentales en pacientes rehabilitados con implantes osteointegrados en clínica privada en el municipio de Vitória-ES. En seguida aparecieron las fracturas verticales radiculares con pino, caries secundarias, fracasos endodónticos, reabsorciones radiculares, traumatismos dentales, otros motivos y fracturas verticales radiculares sin pines. Ningún paciente presentó pérdidas dentales por caries primarias; las razones de las pérdidas dentales mostraron significancia estadística con la variable edad. El sexo, el hábito de fumar y la localización de la pérdida dental no mostraron asociación significativa con las razones de las pérdidas en la clínica privada evaluada.

LOPES, G. R. S.; MATOS, J. D. M.; BARBOSA, G. P. S.; RODRIGUES, A. G.; NISHIOKA, R. S.; ANDRADE, V. C. \& GUERRA, S. M. G. Etiology of tooth loss in patients rehabilitated with dental implants. Int. J. Odontostomat., 12 (3):280-286, 2018.

ABSTRACT: The aim of this study was to analyze the etiology of tooth loss in patients rehabilitated with dental implants. Was conducted a cross-sectional study with data from 93 patients from a private clinic who were treated with implants from the year 2000 until 2009. Data were collected through medical records, radiographic documentation and photographs of clinical cases. Data on sex, age, education level, smoking habits, teeth loss localization and extraction ratio were analyzed. The results showed that periodontal disease was the main reason for tooth loss, accounting for $24.7 \%$, followed by root fractures with posts $(23.7 \%)$ and secondary caries (15.1\%). In patients below 40 years, dental trauma and root fractures were responsible for most of the losses. The reasons for tooth loss showed statistical significance with the age $(p<0.05)$. Sex, smoking habits and education showed no significant association $(p>0.05)$ with the reasons of the tooth loss.

KEY WORDS: etiology, dental implants,tooth loss.

\section{REFERENCIAS BIBLIOGRÁFICAS}

Andersson, B.; Odman, P. \& Carlsson, G. E. A study of 184 consecutive patients referred for single-tooth replacement. Clin. Oral Implants Res., 6(4):232-7, 1995. 
Angelillo, I. F.; Nobile, C. G. \& Pavia, M. Survey of reasons for extraction of permanent teeth in Italy. Community Dent. Oral Epidemiol., 24(5):336-40, 1996

Axelsson, P.; Paulander, J. \& Lindhe, J. Relationship between smoking and dental status in 35-, 50-, 65-, and 75-year-old individuals. J. Clin. Periodontol., 25(4):297-305, 1998.

Bahrami, G.; Vaeth, M.; Kirkevang, L. L.; Wenzel, A. \& Isidor, F. Risk factors for tooth loss in an adult population: a radiographic study. J. Clin. Periodont., 35(12):1059-65, 2008.

Bailit, H. L.; Braun, R.; Maryniuk, G. A. \& Camp, P. Is periodontal disease the primary cause of tooth extraction in adults? J. Am. Dent. Assoc., 114(1):40-5, 1987

Cahen, P. M.; Frank, R. M. \& Turlot, J. C. A survey of the reasons for dental extractions in France. J. Dent. Res., 64(8):1087-93, 1985.

Caldas Junior, A. F.; Silveira, R. C. J. \& Marcenes, W. Impacto do tratamento restaurador na prevenção da perda de dentes. Pesq. Odontol. Bras., 17(2):166-70, 2003.

Cho-Yan Lee, J.; Mattheos, N.; Nixon, K. C. \& Ivanovski, S. Residual periodontal pockets are a risk indicator for peri-implantitis in patients treated for periodontitis. Clin. Oral Implants Res., 23(3):325-33, 2012.

Fardal, Ø.; Johannessen, A. C. \& Linden, G. J. Tooth loss during maintenance following periodontal treatment in a periodontal practice in Norway. J. Clin. Periodontol., 31(7):550-5, 2004.

Heitz-Mayfield, L. J. A. Peri-implant diseases: diagnosis and risk indicators. J. Clin. Periodontol., 35(8 Suppl.):292-304, 2008.

Jovino-Silveira, R. C.; Calda, A. de F. Jr.; de Souza, E. H. \& Gusmão, E. S. Primary reason for tooth extraction in a Brazilian adult population. Oral Health Prev. Dent., 3(3):151-7, 2005

Jovino-Silveira, R. C.; Caldas Júnior, A. F.; de Souza, E. H. A. \& Gusmão, E. S. Razões das perdas dentárias nas cidades de Maceió e Recife, Brasil. Arq. Odontol., 40(3):229-36, 2004.

Karoussis, I. K.; Salvi, G. E.; Heitz-Mayfield, L. J.; Brägger, U.; Hämmerle, C. H. \& Lang, N. P. Long-term implant prognosis in patients with and without a history of chronic periodontitis: a 10year prospective cohort study of the ITI Dental Implant System. Clin. Oral Implants Res., 14(3):329-39, 2003.

Kay, E. J. \& Blinkhorn, A. S. The reasons underlying the extraction of teeth in Scotland. Br. Dent. J., 160(8):287-90, 1986.

Klinge, B.; Hultin, M. \& Berglundh, T. Peri-implantitis. Dent. Clin. North Am., 49(3):661-76, 2005.

Lekholm, U.; van Steenberghe, D.; Herrmann, I.; Bolender, C.; Folmer, T.; Gunne, J.; Henry, P.; Higuchi, K.; Laney, W. R. \& Lindén, U. Osseointegrated implants in the treatment of partially edentulous jaws: A prospective 5-year multicenter study. Int. J. Oral Maxillofac. Implants, 9(6):627-35, 1994.

Lindhe, J.; Meyle, J. \& Group D of the European Workshop on Periodontology. Peri-implant diseases: Consensus Report of the Sixth European Workshop on Periodontology. J. Clin. Periodontol., 35(8 Suppl.):282-5, 2008.

McCaul, L. K.; Jenkins, W. M. \& Kay, E. J. The reasons for the extraction of various tooth types in Scotland: a 15-year follow up. J. Dent., 29(6):401-7, 2001.

Melo, R. E. V. A.; Silva, M. B. L.; Vitor, C. M. A.; Lun, L. A. \& Firmo, A. C. B. Traumatismo dentoalveolar. Int. J. Dent., 2(2):266-72, 2003.

Morita, M.; Kimura, T.; Kanegae, M.; Ishikawa, A. \& Watanabe, T. Reasons for extraction of permanent teeth in Japan. Community Dent. Oral Epidemiol., 22(5 Pt. 1):303-6, 1994.

Ricucci, D. \& Grosso, A. The compromised tooth: conservative treatment or extraction? Endod. Top., 13(1):108-22, 2006.

Roccuzzo, M.; Bonino, L.; Dalmasso, P. \& Aglietta, M. Long-term results of a three arms prospective cohort study on implants in periodontally compromised patients: 10-year data around sandblasted and acid-etched (SLA) surface. Clin. Oral Implants Res., 25(10):1105-12, 2014.
Rokn, A.; Aslroosta, H.; Akbari, S.; Najafi, H.; Zayeri, F. \& Hashemi, $\mathrm{K}$. Prevalence of peri-implantitis in patients not participating in well-designed supportive periodontal treatments: a crosssectional study. Clin. Oral Implants Res., 28(3):314-9, 2017.

Sardinha, S. C. S.; Viana, P. R. L.; Azoubel, E. \& Pinheiro, B. R. G. Levantamento epidemiológico realizado na clínica de cirurgia bucal. Rev. Odontol Ciênc., 21(53):227-31, 2006.

Sgolastra, F.; Petrucci, A.; Severino, M.; Gatto, R. \& Monaco, A. Periodontitis, implant loss and peri-implantitis. A meta-analysis. Clin. Oral Implants Res., 26(4):e8-e16, 2015.

Sulzer, T. H.; Bornstein, M. M. \& Buser, D. Indications for oral implantology in a referral clinic. A three-year retrospective analysis of 737 patients with 1176 implants. Schweiz. Monatsschr. Zahnmed., 114(5):444-50, 2004.

Swierkot, K.; Lottholz, P.; Flores-de-Jacoby, L. \& Mengel, R. Mucositis, peri-implantitis, implant success, and survival of implants in patients with treated generalized aggressive periodontitis: 3- to 16-year results of a prospective long-term cohort study. J. Periodontol., 83(10):1213-25, 2012.

Tonetti, M. S.; Steffen, P.; Muller-Campanile, V.; Suvan, J. \& Lang, N. P. Initial extractions and tooth loss during supportive care in a periodontal population seeking comprehensive care. J. Clin. Periodontol., 27(11):824-31, 2000.

Wegner, P. K.; Freitag, S. \& Kern, M. Survival rate of endodontically treated teeth with posts after prosthetic restoration. J. Endod., 32(10):928-31, 2006.

Willershausen, B.; Tekyatan, H.; Krummenauer, F. \& Briseño Marroquin, B. Survival rate of endodontically treated teeth in relation to conservative vs post insertion techniques -- a retrospective study. Eur. J. Med. Res., 10(5):204-8, 2005.

Dirección para correspondencia:

Guilherme da Rocha Scalzer Lopes

Programa de Doctorado

Departamento de Prostodoncia

Universidade Estadual Paulista

Júlio de Mesquita Filho UNESP

São José dos Campos - SP

BRASIL

Email: matosjefferson19@gmail.com

Recebido : 06-05-2018

Aceptado : 15-06-2018 